\title{
Ugo Foscolo: il classicismo come lirismo
}

\author{
Mariagiovanna LAURETTA \\ Università di Pisa \\ mariagiovanna.lauretta@gmail.com
}

Recibido: 11 de junio de 2014

Aceptado: 5 de julio de 2014

\section{SINOSSI}

Il presente studio propone una lettura selettiva della produzione poetica di Ugo Foscolo alla luce della tradizione classica. L'analisi si è rivolta all'esegesi dei più noti sonetti, del carme Dei Sepolcri, dell'incompiuto lavoro intitolato alle Grazie mentre, solo cursoriamente, si è presa in considerazione l'ode All'amica risanata. Non si è in alcun modo preteso di redigere una compilazione esaustiva di tutti i prestiti classici che affioravano, sia a livello linguistico che tematico, già ad una prima lettura. Si è cercato, invece, di costruire un percorso che, indicando con puntualità l'incidenza del mondo latino (soprattutto Virgilio, Catullo, Lucrezio) e greco (in massima parte Omero) nella poesia foscoliana ed evidenziando come non si tratti in nessun caso di una ripresa meccanica, retorica ed ornamentale, rendesse conto del significato culturale dell'opzione classicista del poeta. Si è, infine, avanzata una proposta ermeneutica che prova a leggere il classicismo di Foscolo come eresia lirica.

Parole chiave: Foscolo. Classicismo. Mito. Lirismo. Tradizione.

Lauretta, M., «Ugo Foscolo: il classicismo come lirismo», Cuad. Fil. Clás. Estud. Lat. 34.2 (2014) $337-358$.

\section{Ugo Foscolo: Clasicism as Lyrism}

\begin{abstract}
The purpose of my study is to propose a selective reading of Ugo Foscolo poetry in the light of the classical tradition. My analysis is directed to the exegesis of his best-known sonnets, Dei sepolcri, and the incomplete work, Le Grazie, while I have only marginally evaluated the ode All'amica risanata. I do not in any way claim to draw up a comprehensive compilation of all classic loans that cropped up, both linguistically and thematically, since the very first reading. I have tried, however, to build a path that realize the cultural significance of the classicistic option of the poet, pointing with punctuality the incidence of the Latin (especially Virgil, Catullus, Lucretius) and the Greek world (mostly Homer) in Foscolo poetry highlighting as in no case it is a mechanic, rhetoric and ornamental retake. I have finally promoted a hermeneutical proposal that tries to read the classicism of Foscolo as the result of his lyrical intent.
\end{abstract}

Keywords: Foscolo. Clasicism. Myth. Lyrism. Tradition.

Lauretta, M., «Ugo Foscolo: Clasicism as Lyrism», Cuad. Fil. Clás. Estud. Lat. 34.2 (2014) 337-358.

Sommario: 1. Introduzione. 2. I sonetti. 3. Il carme Dei Sepolcri. 4. Le Grazie. 5. Conclusioni. 6. Bibliografia. 


\section{INTRODUZIONE}

Delineare il percorso della produzione foscoliana alla luce della tradizione classica -in tutte le sue accezioni- è operazione essenziale per cogliere pienamente la portata dell'operato poetico e civile dell'autore. Sebbene a lungo si sia voluto leggere il classicismo foscoliano come esito del suo essere nativo di terra greca -l'isola di Zante la quale si trovava, nel 1817, sotto il protettorato di Venezia- e dell'essere figlio di madre greca, in tempi più recenti si è dimostrato essenziale il rapporto che lega il poeta al ritorno neoclassico di tanta cultura, italiana e non, della seconda metà del '700. Epperò l'esito foscoliano di miti, temi, termini o semplici ritmi classici è affatto originale, viscerale quasi, per l'insistenza produttiva di due tensioni distinte ma cooperanti, sintetizzabili come polarità individuale-collettivo ${ }^{1}$ : da un lato la natura soggettiva inquieta e sempre anelante ad un misurato equilibrio, dall'altro il senso di una obbligazione etica, costantemente alimentato dal momento storico d'Italia e d'Europa, l'una e l'altro confluendo in una coazione estetica di natura, come vedremo, mitopoietica.

A voler tracciare una parabola dei classici assunti come riferimento da Foscolo, risulta perspicuo un fatto non liquidabile come mera cronologia relativa: una prima fase della sua produzione -che è arduo definire giovanile se si assume il carme Dei Sepolcri come spartiacque- vede una presenza maggioritaria dei latini (Tibullo, Catullo, Orazio, Tacito, Virgilio fino al Lucrezio del carme) a livello linguistico, mentre il mondo greco sembra connotato come fecondo e immediato bacino immaginifico. Un dato, questo, in apparenza straniante ove si consideri che Foscolo per sua stessa ammissione «era appena tinto della lingua latina [...] quando venne di Grecia in Italia» (Fubini 1972, p.239) e che tuttavia acquista pregnanza alla luce della relazione, dinamica ed evolutiva, che il poeta intrattiene con gli universi greco e latino. Il senso incipitario di tale relazione risolve il mondo greco come repertorio mitico, dizionario di segni plastici che in questa fase non convergono ancora in una affabulazione coerente, ma funzionano come deissi di un nodo esistenziale critico, mentre del mondo latino Foscolo pare ricercare la qualità espressiva, l'orizzonte formale capace di mediare temi e motivi attinti direttamente dai greci. In questa fase aurorale della poetica di Foscolo i latini sono dunque un banco di prova, un tirocinio linguistico e stilistico, un laboratorio alla ricerca di una possibilità di storicizzazione, mediante la sperimentazione verbale, dell'istinto mitico nativo. Il poeta si prova nel dominio dei due orizzonti, ancora e a lungo distinti quand'anche non alternativi, talora sovrapponibili ma mai interscambiabili perché distinta è la funzione mentale ed intellettuale a

\footnotetext{
${ }^{1}$ Per il senso dell'interferenza individuale-collettivo e della incidenza solidale di questi elementi sulla produzione culturale umana, si rimette alla felice formulazione dell'idea di «uomo totale» elaborata dall'etnologo Marcel Mauss, ad intendere la complessità fisiologica e psicologica dell'uomo. Caratterizzata da un livello di corporeità e da un livello psicologico complesso in cui si obiettivano istanze singolari e collettive, la persona umana, nella determinazione delle proprie azioni morali -ovvero i comportamenti-e di quelle culturali -vale a dire le opere- riflette non solo suggestioni individuali ma anche sociali. Cf. Mauss 1950, passim.
} 
cui rispondono: una cesura che solo la scelta conclusiva del lirismo mitopoietico sarà in grado di sussumere.

In ragione di quanto detto, assumo l'ordine cronologico quale valido criterio ordinativo e descrittivo della produzione foscoliana, nella consapevolezza che non può trattarsi di una chiave ermeneutica esaustiva giacché non risponde, da un punto di vista genetico e ontologico, al modus operandi di un poeta che si impegnó contemporaneamente e a pieno ritmo su fronti diversissimi: dalle traduzioni, agli studi di interesse erudito, alla stesura di liriche e prose di natura assai eterogenea. L'ordinamento cronologico aiuterà certo il lettore a discernere, per entro il tumulto vorticoso della riflessione sulla realtà e della sua manipolazione e trasfigurazione formale, il filo rosso di una decisiva intenzione poetica.

La nostra breve analisi si dirigerà, anzitutto, ai più noti sonetti, esaminerà brevemente il momento di transizione cruciale rappresentato dal carme Dei Sepolcri e si concluderà con l'Inno alle Grazie che -in quanto risultato di un rimaneggiamento di cinque frammenti di un poema il cui primo nucleo fu ideato nel 1803 e mai portato a termine- può a buon diritto ritenersi l'emblema del classicismo di Foscolo, il quale nel suo tradere il mondo greco e latino si convertì in traditore più ancora che in traduttore di quei mondi, facendo dell' cik $\alpha \sigma i ́ \alpha$ mitica una proposta di ergatività e riconsegnando a se stesso e ai suoi contemporanei la possibilità di una presenza storica nella dimensione estetica del classicismo.

\section{I SONETTI}

Forse perché della fatal quïete tu sei l'imago a me sì cara vieni o sera! E quando ti corteggian liete le nubi estive e i zeffiri sereni e quando dal nevoso aere inquïete tenebre e lunghe all'universo meni sempre scendi invocata, e le secrete vie del mio cor soavemente tieni.

Vagar mi fai co' miei pensier su l'orme che vanno al nulla eterno; e intanto fugge questo reo tempo, e van con lui le torme

delle cure onde meco egli si strugge; e mentre io guardo la tua pace, dorme quello spirto guerrier ch'entro mi rugge. (Foscolo 1802/3, Alla Sera)

Forse perché della fatal quiete... ${ }^{2}(1802 / 1803)$ è probabilmente il sonetto più noto tra $\mathrm{i}$ versi foscoliani. Il primo elemento da considerare per l'esegesi del com-

\footnotetext{
${ }^{2}$ Il sonetto ha ricevuto il titolo postumo di Alla sera.
} 
ponimento è un dato di natura extratestuale, ovvero la circostanza che il sonetto, in una prima redazione, fu rinvenuto nel volume del De rerum natura che Foscolo stava maneggiando evidentemente in vista di una traduzione di Lucrezio. L'influenza del materialista latino non può non saltare all'occhio già nell'equivalenza che il poeta istituisce tra sera, quiete, morte, immediatamente riconducibile alla riflessione lucreziana sulla morte, la quale, in ragione della sua somiglianza con il sonno ed il riposo, non può dirsi condizione che incuta paura; al poeta-filosofo rimandano anche le due terzine conclusive. Persino il «nulla eterno», alla luce della filosofia lucreziana, non induce al timore: è solo il tempo che precede e segue alla morte. Indubbiamente, se il tempo è edax nella lectio dei classici (Hor.Carm1.11.7-8: Dum loquimur/fugerit inuida aetas), primo fra tutti; però anche VERG.Georg.3.284: sed fugit interea, fugit inreparabile tempus), in questi versi il tema della fuga si carica di un significato positivo; l'aggettivo «reo» (acerbus nel saggio su Lucrezio) assicura in certa misura il recupero di questa accezione positiva, vincolata però alla condizione del distacco, della separazione: lo «spirto guerrier» «dorme», vale a dire si acquieta a condizione che il «reo tempo» e con lui «le cure» fuggano, si dissipino, permettendo al poeta quella meditazione che conduce al «nulla eterno». Ė questa la lezione di Lucrezio nel De rerum natura (LuCR.2.1-19).

A proposito del termine «cura», importa qui evidenziare come del sostantivo Foscolo si avvalga con frequenza -in accezioni diverse, ma tutte congruenti- sia nel commento a La chioma di Berenice (opera degli stessi anni, pubblicata presso l'editore Agnello Nobile di Milano insieme alle Poesie in cui Alla sera compare come sonetto proemiale), sia nelle prime stesure dell'Ortis («come sepolto in tristissime cure»), sia, infine, nella lettera a G. Rosini del 9 gennaio 1803 («morior curis»). In generale, non è scorretto affermare che «cura» sembra ampliare il suo significato, da «prepotente desiderio che vive in noi, pieno di speranze e di timori ${ }^{3}$ » fino a denotare una condizione di melancolia in senso lato, e tuttavia non meno intenso: un binomio di significati non esattamente antitetici, ma piuttosto irriducibili a complementarietà, che sintetizza bene il sentimento di Foscolo di fronte ai temi esistenziali sui quali si sottopone a costante interrogazione.

È doveroso, certo, non tacere la massiccia presenza dei classici latini nelle quartine: al di là delle scelte lessicali di gusto eminentemente etimologico ${ }^{4}$, è Virgilio chi risulta estremamente presente nei versi 1-8; gli «zaffiri sereni» rimandano con puntualità alle serenas nubes delle Georgiche, mentre dell'Eneide Foscolo sembra mutuare l'individuazione delle notti lunghe per contrassegnare l'inverno (VERG. Aen.1.745-746); sempre al poema epico fa eco la visione enfatica dei versi 5-6 (VERG. Aen.2.250-251: nox/inuoluens umbra magna terramque poluumque).

La trama sotteranea, il substrato direi, del classicismo tardo-settecentesco consarcina la atmosfera portante del sonetto: Foscolo non nega un tributo al gusto neoclassi-

\footnotetext{
${ }^{3}$ Così ne La chioma di Berenice.

${ }^{4}$ Solo per fare qualche esempio: lieto da laetus, è aggettivo che i latini amavano applicare alla natura; inquiete, etimologicamente in-quiete, è precisamente «senza pace».
} 
co del suo tempo nella scelta di una armonia e una misura generali che, soprattutto a partire dal livello formale delle singole scelte lessicali, ricompongono una immagine della natura -e per via immaginativa si realizza anche la ricomposizione-istituzione del soggetto lirico- accorde alle prospettive neoclassiche di matrice winckelmaniana.

Virgilio ma anche Catullo e Tibullo ritornano come modello nel sonetto In morte del fratello Giovanni, suicida l'8 dicembre 1801 in circostanze misteriose. Composto per ultimo e pubblicato solo nell'edizione definitiva delle Poesie (quella già citata di Nobile, cf. supra, p.340), conobbe probabile ispirazione occasionale nella rilettura del carme 101 di Catullo, che dovette indurre in Foscolo una tensione comparativa, l'esigenza di raffrontare il proprio stato d'animo con quello del poeta antico.

Si riproducono di seguito $i$ due testi menzionati, perchè la comparazione sia di più agile lettura.

Un dì, s'io non andrò sempre fuggendo

di gente in gente, me vedrai seduto

su la tua pietra, o fratel mio, gemendo

il fior de' tuoi gentil anni caduto.

La Madre or sol suo dì tardo traendo

parla di me col tuo cenere muto,

ma io deluse a voi le palme tendo

e sol da lunge i miei tetti saluto.

Sento gli avversi numi, e le secrete cure che al viver tuo furon tempesta, e prego anch'io nel tuo porto quiete.

Questo di tanta speme oggi mi resta!

Straniere genti, almen le ossa rendete allora al petto della madre mesta. (Foscolo 1803, In morte del fratello Giovanni)

\author{
Multas per gentes et multa per aequora uectus \\ aduenio has miseras, frater, ad inferias, \\ ut te postremo donarem munere mortis \\ et mutam nequiquam alloquerer cinerem. \\ quandoquidem fortuna mihi tete abstulit ipsum. \\ heu miser indigne frater adempte mihi, \\ nunc tamen interea haec, prisco quae more parentum \\ tradita sunt tristi munere ad inferias, \\ accipe fraterno multum manantia fletu, \\ atque in perpetuum, frater, aue atque uale. (Catullo, Carmina, 101)
}

L'incipit catulliano offre evidentemente l'attacco al sonetto foscoliano, ma la distanza dal modello è subito patente nel desiderio respinto della visita al defunto, improbabile all'inizio e in conclusione dichiaratamente irrealizzabile, in ragione della condizione d'esiliato tra «straniere genti». 
I motivi comuni -il dolore, il molto viaggiare, il dialogo che si fa monologo perché i resti (cinerem) sono muti (mutum) - sono, a ben vedere, segni più di dissociazione che di comunione empatica.

Come ha ben evidenziato Vincenzo Di Benedetto (1990, pp.34 e sgg.) nel carme catulliano il poeta conserva la possibilità di agire un rito, il compimento del quale garantisce al vivo la reintegrazione a dispetto della sua condizione. Quel tamen (v. 7) sembra proprio il centro logico del carme visto che esplicita la possibilità, la opportunità che il poeta ha, nonostante tutto, di ricongiungersi al fratello: egli, vivo, reduce da gentes ed aequora, parla al defunto e a lui tributa la sua parte, un ultimo munus mortis; cosí, il fratello morto è solo in apparenza mutum cinerem, e solo in apparenza nequiquam registra in forma indefinita la frattura definitiva tra vita e morte. Quel tamen allarga il confine tra le due condizioni e disegna uno spazio, un luogo d'incontro -la tomba- in cui l'uno, Catullo, offre e l'altro, il fratello, riceve la parte che gli spetta (l'accipe in imperativo presente, connota di certezza l'accoglimento della prestazione rituale da parte del defunto molto più di quanto non avrebbe potuto un congiuntivo desiderativo). La frattura temporale pare superata dall'in perpetuum del v. 10, mentre il uale conclusivo sembra risanare la linea interrotta dalla morte.

Non così in Foscolo, per il quale «l'andare di gente in gente costituisce un movimento centrifugo che lo allontana dal fratello» (Di Benedetto 1990, p.35).

Forse è proprio per questo, perché a lui è negato il compimento del rito, che il poeta di Zante affida il suo canto all'indole nostalgica dell'elegiaco Tibullo; a lui rimandano, infatti, i versi incipitari e quelli conclusivi del sonetto: illius ad tumulum fugiam supplexque sedebo (Тiв.2.6.33-34) ${ }^{5}$. Ancora, i versi 13-14 del poema sono eco dei tibulliani non hic mihi mater/quae legat in maestos ossa perusta sinus (Tів.1.3.56). L'ambito degli affetti familiari è dunque il terreno di incontro di Foscolo, Catullo e Tibullo, accomunati dal lutto fraterno o sororale, ma separati dalla speranza di un ricongiungimento nel rito.

Un'altra fonte alquanto esplicita del carme è, ancora una volta, il Virgilio dell'Eneide. «Il fior de' tuoi gentili anni caduto» ricorda da vicino la famosa similitudine catulliana uelut prati /ultimi flos, praetereunte postquam / tactus aratro est (CATULL.11.22-24), che Virgilio rimodula in ueluti cum flos succisus aratro / languescit moriens (VERG.Aen.9.435-436); ancora Virgilio ritorna al v. 7 inualidas tibi tendens / heu non tua palmas (VERG.Georg.4.498), e al v. 9 conuersaque numina sentis (VERG. Aen.5.466).

Mi pare, tuttavia, che i tentativi arditi operati da Foscolo nel modellare la sua lingua su quella degli antichi -che pure mostrano esiti notevoli- passino in secondo piano rispetto all'impianto complessivo del testo, al tono prevalente, tutto elegiaco, che permea il luogo sacro degli affetti familiari: la tomba come il testo. Torna in mente quel «cure» di cui già si è parlato, la cui funzione emotiva è qui declinata dall'aggettivo «secrete», dagli «avversi numi», dalla «tempesta del viver», tutti nessi

\footnotetext{
${ }^{5}$ La stessa fonte viene riutilizzata da Foscolo nel carme Dei Sepolcri ai versi 126-128. È merito di T. Casini (1891, p.19) aver notato e indicato per primo il doppio parallelo.
} 
che lungi dal generare un'angosciosa disperazione, acquistano misura nella mestizia composta della madre del poeta, cui Foscolo assegna la funzione liminale di sacerdotessa di quel rito il cui accesso è a lui vietato: la madre parla (v. 6), la madre accoglie (vv. 13-14), la madre compie il rito.

Il tema dell'esilio e la figura materna dominano il sonetto dedicato alla greca isola natia, Zacinto, e costituiscono il fulcro dell'ispirazione tutta virgiliana della produzione foscoliana di questi anni.

Né più mai toccheró le sacre sponde ove il mio corpo fanciulletto giacque, Zacinto mia, che te specchi nell'onde del greco mar da cui vergine nacque

Venere, e fea quelle isole feconde col suo primo sorriso, onde non tacque le tue limpide nubi e le tue fronde l'inclito verso di colui che l'acque cantò fatali, ed il diverso esiglio per cui bello di fama e di sventura baciò la sua petrosa Itaca Ulisse.

Tu non altro che il canto avrai del figlio, o materna mia terra; a noi prescrisse il fato illacrimata sepoltura. (Foscolo 1803, A Zacinto)

Se è certamente vero che ad una prima lettura si ha l'impressione che a dominare il testo sia la suggestione omerica e specificamente odissiaca -al poeta epico Foscolo si richiama esplicitamente al v. 8 («colui che l'acque cantò fatali») e non manca di riconoscergli indiscutibile gloria poetica («inclito verso»)- non sfugge ad un' analisi più accorta, tanto delle scelte lessicali e linguistiche come delle immagini paesaggistiche, l'ordito virgiliano che opera a un livello più profondo della trama poetica.

Ancora una volta si deve a Di Benedetto l'enucleazione, nel sonetto, di plurime suggestioni virgiliane e insieme l'identificazione del senso dell'incipitaria ispirazione omerica. Pare a me che il richiamo ad Omero sia precisamente una mozione dello spirito, un moto intuitivo dell'intelligenza fantastica che attinge all'immagine mitica dell'eroe epico nella misura in cui vi riconosce un modello iconico universale e metastorico, con il preciso intento di tipizzarlo, di defraudarlo della sua identità mitica greca e riplasmarlo, attraverso l'azione mediatrice dell'alter ego latino Enea, nella dimensione biografica e storica dell'io lirico del poeta (Salvini 2003, pp.71-72). Il paesaggio, dicevamo, prepara questo slittamento: alla «petrosa Itaca» baciata dall'eroe al suo ritorno, immagine evidentemente mutuata dal libro XIII dell'Odissea (vv. 353-354), Foscolo accosta le «le limpide nubi» in associazione con le «fronde», il poeta costruisce, cioè, una natura squisitamente virgiliana (VERG.Aen.5.525: liquidis in nubibus). 
Si proietta su uno sfondo siffatto, la figura tutta nuova di un Ulisse che poco o nulla reca in sé dell'astuto eroe odissiaco e piuttosto svela nel suo «diverso esiglio» su «acque fatali» quella medesima ascendenza latina che si è intravista nel carme In morte del fratello Giovanni. I due testi foscoliani sono, come si è detto, intimamenti connessi, anzitutto per l'allusione diretta al senso del proprio ruolo poetico che pare prodotto resultativo della condizione del molto viaggiare, di quell'esilio che impedisce a Foscolo di sedersi sulla tomba del fratello e che tuttavia gli garantisce una implicita investitura poetica nel destino che lo accomuna al più famoso degli aedi dell'antichità; nell'ultima terzina di A Zacinto, in particolare, l'esercizio della funzione poetica è l'ultimo atto etico disponibile a chi è costretto dalla politica all'allontanamento coatto, ed il poeta sembra ora voler asseverare la propria presenza storica nel non luogo, nel non tempo melici della rapsodia.

In secondo luogo, il richiamo alla «materna... terra», vagheggiata ma irraggiungibile, rimanda -in una lettura dei contenuti allusi dal codice dell'inconscio- ad una immagine nitida di grembo materno e nascita, certo assimilabile a quella della madre che, sulla tomba, è preposta ad accogliere «le ossa...rese...da straniere genti».

Alla luce di queste considerazioni non stupisce allora che Odisseo diventi prima Ulisse e poi il poeta stesso attraverso la mediazione di Enea, la cui figura Foscolo innesta dentro questo mito-biografia a più riprese: già con il «diverso esiglio» che il poeta traduce dal III libro dell'epica virgiliana (VERG.Aen.3.4: diversa exsilia), fino allo stilema binomiale «bello di fama e di sventura» che ritroviamo più volte nel poema, diversamente declinato secondo la logica di variazione/ripetizione che presiede alla dizione formulare (Cf. e.g. VERG.Aen.5.295: Euryalus forma insignis uiridique iunenta; o ancora Aen.7.745: insignem fama et felicibus armis $)^{6}$.

La connotazione virgiliana di Ulisse si intende, una volta di più, alla luce della percezione che Foscolo ha del proprio destino di esiliato che, da un parte, lo induce ad ancorare la natura eroica al rientro in patria (elemento, questo, che in Omero non ha affatto carattere esiziale), dall'altra, lo spinge a riformulare i connotati dell'eroicità -come era avvenuto anche nel sonetto per il fratello- come conseguenza della «dura prescrizione del fato», nella cui consapevole accettazione il poeta rivendica orgogliosamente un marchio eroico.

Si noti, infine, che l'evocazione di Zacinto, funzionale al mito della predestinazione poetica, è realizzata in modo affatto originale mediante la posizione di una serie analogica che spazia dalla geografia reale a quella mitica: Zacinto si specchia nelle onde del mare che vide la nascita di Venere, la dea che illuminò del suo sorriso quelle isole al punto che Omero fu indotto a cantarne la bellezza, rammemorando l'avventuroso viaggio di Ulisse destinato, infine, ad approdare ad Itaca. Zacinto diviene così non tanto e non solo un luogo della memoria, ma il luogo del mito, della poesia, uno spazio astorico in cui la personalità del poeta si riavvicina alla dimensione del vitale, della morale. A sorreggere questa costruzione, l'apparato sintattico

\footnotetext{
${ }^{6}$ È opportuno rilevare che Foscolo risemantizza lo stilema binario con la sostituzione, al secondo elemento, di un termine positivo con uno negativo.
} 
si modula in una immane struttura copulativa: tutte le proposizioni legate attraverso connettori relativi, che si dipanano l'uno dall'altro e dentro l'altro («ove...che...da cui... onde...che...per cui»). È questa la via, precisamente mitopoietica, che Foscolo sceglie per costruire la propria biografia poetica, installandola saldamente su una geografia favolosa che vede Venere nata nel mar Ionio. Già Esiodo nella Teogonia (HEs.Th.190-193) aveva affermato che Afrodite «prima si avvicinò alla divina Citera, e di lí giunse poi a Cipro circondata dal mare», e ora Foscolo manipola il racconto epico e lo rimaneggia così da tacere Citera ed enfatizzare il mare, lo Ionio dalle acque feconde per il sorriso della dea ${ }^{7}$.

Con questo virtuoso gioco di allusioni Foscolo riesce a riconnettere Zacinto a Omero e a realizzare compiutamente la circolarità del testo: l'apostrofe a Zacinto in apertura e in chiusura, insieme all'opposizione tra l'immagine della prima infanzia e quella della sepoltura (vv. 2 e 14), racchiudono il paradigma di una esistenza intera.

A partire da questo momento e costantemente, la geografia diverrà solo vago riferimento, e quando il poeta porrà mano alla stesura del carme Dei Sepolcri potrà già giovarsi della sua propria riscrittura geo-storica degli antichi racconti mitici; affiderà così alla propria riplasmazione delle immagini mitiche tradizionali, alla propria affabulazione -che com'è s'è visto è ad un tempo geografia mito e biografia- una funzione strutturale capace di garantire unitarietà noetica agli impianti dispersivi e «pindarici» che caratterizzeranno tanto il carme come l'Inno alle Grazie.

Per concludere l'analisi sul «discorso geografico» di Foscolo, vale la pena soffermarsi, pure desultoriamente, sull'operazione, affatto simile a quella vista finora, che il poeta compie nell'ode All'amica risanata, la cui stesura risale al 1802, ad un momento, dunque, del tutto contiguo a quello della composizione dei sonetti.

Qual dagli antri marini

l'astro più caro a Venere

co' rugiadosi crini

fra le fuggenti tenebre

5 appare, e il suo viaggio

orna col lume dell'eterno raggio

[...]

E quella a cui di sacro

mirto te veggo cingere

75 devota il simolacro,

che presiede marmoreo

agli arcani tuoi lari

ove a me sol sacerdotessa appari,

regina fu; Citera

80 e Cipro ove perpetua

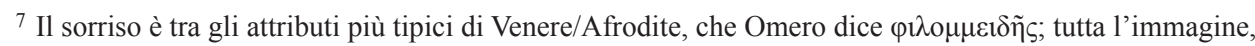
poi, richiama da vicino l'incipit del poema sulla natura lucreziano (Cf. LuCR.1.2-9).
} 
odora primavera

regnò beata, e l'isole

che col selvoso dorso

rompono agli euri e al grande Ionio il corso.

85 Ebbi in quel mar la culla,

ivi erra ignudo spirito

di Faon la fanciulla,

e se il notturno zeffiro

blando su i flutti spira,

90 suonano i liti un lamentar di lira.

Ond'io, pien del nativo

aër sacro, su l'Italia

grave cetra derivo

per te le corde eolie,

95 e avrai, divina, $i$ voti

fra gl'inni miei delle insubri nipoti. (Foscolo 1802, excerpta da All'amica risanata)

Anche in questo caso la geografia assume connotati personali e identitari, e Zacinto viene impreziosita quale luogo privilegiato del mito e dell'arte, rappresentazione icastica della funzione eternatrice del poeta.

Nell'ode, che si apre con l'immagine di Espero che sorge tra le tenebre della not$t^{8}$, Foscolo fa leva sull'elemento liquido del passaggio, il mar Ionio, per far affiorare il dato della sua nascita a Zacinto. I versi a cui ci riferiamo seguono alla rassegna delle donne mortali divinizzate: Artemide, Bellona, Venere. Quest'ultima deificazione consente al poeta di inaugurare un articolato paragone: la dedicataria dell'ode viene avvicinata a Venere, alla quale offre doni votivi nella veste di sua sacerdotessa, mentre il poeta diviene novello Saffo, la nascita comune nel mare greco a garantire la legittimità dell'assimilazione. Per denotare il senso del legame continuativo con e tra le isole ionie (e dunque stabilire l'equazione Leucade-Saffo, Citera-Venere) il poeta rifugge dall'evocazione onomastica di Zacinto e individua nel mare l'elemento connettivo aggregante; con «ebbi in quel mar la culla» (v. 85) e subito dopo con «ivi» (v. 86) Foscolo evita di fissare un luogo specifico e stabile tra quelle terre e assegna al mare il primato geografico e soprannaturale della poesia.

Nei versi finali, la memoria foscoliana tradisce ancora non un poeta greco, ma latino. È l'Orazio del «Congedo» del terzo libro delle odi (HoR.Carm.3.30.10-14), che era già apparso nell'immagine della lira di Saffo che risuona ancora nel mare delle isole ionie: spirat adhuc amor / uiuntque comissi calores / Aeoliae fidibus puellae. Il mare assume così definitivamente un valore ideale, soprannaturale, metastorico, stigma simbolico che oggettiva la radice della vocazione poetica: Foscolo, come Saffo,

\footnotetext{
${ }^{8}$ Ancora una volta l'immagine è virgiliana (VERG.Aen.8.586-591) e, possiamo dirlo, pare proprio che il poeta italiano intessa con Virgilio una relazione filogenetica costitutiva del suo fare poetico.
} 
avrà il potere di eternare l'amica. Sempre al mondo latino è debitrice l'idea del valore immortalizatore della parola poetica; basti, in questa sede, menzionare il Properzio elegiaco che, nel passare in rassegna le molte donne che ebbero fama per merito dei poeti, esprime la propria speranza di entrare nella schiera dei rapsodi, ove la fama glielo conceda: Cinthia quin etiam versu laudata Propertii hos inter si me ponere fama volet (PROP.2.34).

\section{IL CARME DEI SEPOLCRI}

Felice incontro tra la tradizione latina e l'ispirazione neoclassica e protoromantica, il carme Dei Sepolcri traduce il senso della tensione lirica di Foscolo, cantore ora di un eroismo politicamente sconfitto e tuttavia immortale nella dimensione etica della mitopoiesi.

235 Ed oggi nella Troade inseminata eterno splende a' peregrini un loco, eterno per la Ninfa a cui fu sposo Giove, ed a Giove diè Dàrdano figlio, onde fur Troia e Assàraco e i cinquanta

240 talami e il regno della giulia gente. Però che quando Elettra udí la Parca che lei dalle vitali aure del giorno chiamava a' cori dell'Eliso, a Giove mandò il voto supremo: -E se, diceva,

245 a te fur care le mie chiome e il viso e le dolci vigilie, e non mi assente premio miglior la colontà dÈ fati, la morte amica almen guarda dal cielo onde d'Elettra tua resti la fama.-

250 Così orando moriva. E ne gemea l'Olimpio: e l'immortal capo accennando piovea dai crini ambrosia su la Ninfa, e fe' sacro quel corpo e la sua tomba. Ivi posò Erittonio, e dorma il giusto

255 cenere d'Ilo; ivi l'iliache donne sciogliean le chiome, indarno ahi! deprecando da' lor mariti l'imminente fato; ivi Cassandra, allore che il Nume in petto la fea parlar di Troia il dí mortale,

260 venne; e all'ombre cantò carme amoroso, e guidava i nepoti, e l'amoroso apprendeva lamento a' giovinetti. E dicea sospirando:- Oh se mai d'Argo, ove il Tidíde e di Läerte al figlio

265 pascerete i cavalli, a voi permetta 
ritorno il cielo, invan la patria vostra cercherete! Le mura, opra di Febo, sotto le lor reliquie fumeranno.

Ma i Penati di Troia avranno stanza

270 in queste tombe; ché dÈ Numi è dono servar nelle miserie altero nome.

E voi, palme e cipressi che le nuore piantan di Priamo, e crescerete ahi presto

di vedovili lagrime innaffiati,

275 proteggete i miei padri: e chi la scure asterrà pio dalle devote frondi men si dorrà di consanguinei lutti, e santamente toccherà l'altare. Proteggete i miei padri. Un dí vedrete

280 mendico un cieco errar sotto le vostre antichissime ombre, e brancolando penetrar negli avelli, e abbracciar l'urne, e interrogarle. Gemeranno gli antri secreti, e tutta narrerà la tomba

285 Ilio raso due volte e due risorto splendidamente su le mute vie per far più bello l'ultimo trofeo ai fatali Pelídi. Il sacro vate, placando quelle afflitte alme col canto,

290 i prenci argivi eternerà per quante abbraccia terre il gran padre Oceàno. E tu onore di pianti, Ettore, avrai, ove fia santo e lagrimato il sangue per la patria versato, e finché il Sole

295 risplenderà su le sciagure umane. (Foscolo 1807, Dei Sepolcri, vv. 235-290)

Per dischiudere il significato del carme, mi pare ragionevole seguire la linea interpretativa che individua come antipolari il tema dell'immortalità offerta dall'arte poetica e il materialismo meccanicista di matrice lucreziana.

L'incipit del poema è all'insegna della negazione: l'istanza di sopravvivenza etica dell'uomo, che nel sepolcro pare trovare appagamento, è subito vanificata entro la visione di quella distruzione cosmica che è legge dell'universo. La vita, o meglio ancora il principio di vitalità, è fissata in tre immagini di stampo classico, tra le quali primeggia, e non solo in virtú dell'ordine sequenziale, quella desunta dai versi incipitari del De rerum natura: 1' «ove più il sole / per me alla terra non fecondi questa / bella d'erbe famiglia e d'animali» corrisponde esattamente al per te quoniam genuus omnia animantum / concipitur uisitque exortum lumina solis ...tellus lucreziano (LuCR.1.4-8). A questa segue l'immagine delle «ore danzanti» che -già usata da Foscolo in All'amica risanata - ha origini antiche: si trova già in Omero (Hom.Il.5.740751) e poi nell'Inno callimacheo ad Apollo (CALL.Ap.194-199). 
La vita, dicevamo, è l'unico valore, e la morte l'assoluta disgregazione; ancora una volta Lucrezio è la fonte di Foscolo: i versi 19-22 «e una forza operosa le affatica di moto in moto / e l'uomo e le sue tombe e l'estreme sembianze e reliquie / della terra e del ciel traveste il tempo» riproducono da vicino il lucreziano omnia migrant / omnia commutat natura et uertere cogit (LUCR.5.830-831). E tuttavia all'interno di questo andamento ciclico che alterna la vita alla morte Foscolo inserisce un punto di vista nuovo che, lungi dal respingere l'assioma materialista appena formulato, prende avvio da una sostituzione di ordine logico e psicologico che abolisce il tempo umano come valore ontologico e fondativo dell'esistenza. Se il verso 22 riconosce la supremazia del tempo sull'esistente, sul vitale, già il verso successivo («Ma perché pria del tempo») introduce la nuova prospettiva: esistono un prima e un dopo il tempo storico, non vitali epperò cronologici, sull'asse dei quali l'uomo può proporre un ethos: quello astorico della poesia. Lo spazio, «il limitar di Dite» (v. 25), entro cui l'uomo deprivato della sua vitalità, il morto, può operare questo riscatto, è la tomba, attorno alla quale i vivi compiono quella rituale rammemorazione che riafferma la natura trascendente dell'esistenza umana. La poesia, dunque, è un enunciato etico formalmente costruito come una cerimonia religiosa e, nella misura in cui è metastorica acquista la forza cogente dell'emulazione. Non stupisce affatto che Foscolo dispieghi nel carme la più potente delle sue plasmazioni mitiche, giacchè il mito «in quanto espressione spontanea della immaginazione naturale e del sentimento religioso (...) raggiunge il massimo grado di creatività, sia per la produzione di immagini divine che per il valore pedagogico-politico» (Salvini 2003, p.78).

La selezione foscoliana della tradizione classica si fa a questo punto più tematica che filologica; se i moduli linguistici sono comunque presenti, essi costituiscono non una istanza sperimentale bensì una base ormai consolidata nella poesia di Foscolo. È ora il poeta stesso -nelle sue note- a segnalare i prestiti desunti dagli antichi. Cita così, tra le sue fonti, oltre a Lucrezio da cui attinge a piene mani (e.g. v. 44 da Rer. nat.3.85-86), anche Virgilio (vv. 98-99 da Aen.3.62 e sgg.; vv. 258-259 da Aen.3.246247), Persio (vv. 124-125 da Sat.1.38), Tibullo (v. 126 da Eleg.2.6.33-34), l'Omero dell'Iliade e dell'Odissea (vv. 217-218 da Il.7.86 e sgg. e Od.24.76 e sgg.) e ancora Pindaro (vv. 294-295 da Ist.4.40-44) e Leonida di Taranto (A.P.6.715).

Precisamente perché tanto solido, questo substrato classico è pronto per una nuova costruzione, attraverso un'operazione che Di Benedetto presenta come contaminazione del modello, e che permette a Foscolo di incardinare la sua riflessione sulla dinamica vita-morte alla luce del tratto libertario dischiuso dal destino di morte. È la lezione assoluta dell'Iliade quella che Foscolo recupera e oppone al senso del tempo lucrezianamente inteso; una lezione che implica una alienazione dal tempo storico e consacra invece, per il tramite della morte bella, il tempo lungo della rammemorazione poetica. Gioca in questo un ruolo essenziale quella geografia rivisitata la cui origine si è già decifrata.

Il campo di Maratona, sul quale ogni notte spira il Nume della virtù greca, è lo scenario mitico su cui Foscolo fa campeggiare i protagonisti tràditi di questa sua 
storia dell'immortalità. Si susseguono, pindaricamente, i personaggi della storia e del mito, accomunati, tutti, dalla vittoria sul «silenzio di mille secoli» (v. 234). Quel Nume ridà vita alle battaglie notturne (vv. 201-212) ma soprattutto segnala una nuova forma di eroismo, quello di Aiace, quello di Ettore: «A' generosi / giusta di glorie dispensiera è morte».

In una dimensione profonda, che trascende l'antinomia meccanica tra vincitori e vinti, il destino di morte diviene spazio morale per l'uomo in tanto e in quanto si fa foriero del riscatto del tempo. È Ettore, ben al di sopra dei Prenci Argivi, chi avrà «onore di pianti (...) / ove fia santo e lagrimato il sangue / per la patria versato, e finchè il Sole / risplenderà su le sciagure umane».

È specialmente nell'ultima sezione del carme che Foscolo attiva la sua potente rivisitazione del modello omerico ed è patente nel passo relativo alle tombe di Elettra e di Ilo.

Nel segmento conclusivo del poema (vv. 235-295) sono, infatti, ancora i luoghi omerici a fornire la materia del canto, e se nella parte immediatamente antecedente erano i luoghi di sepoltura degli eroi greci ad essere ricordati, sono ora i vinti troiani l'oggetto della rammemorazione lirica. La loro terra, la «Troade inseminata», è ormai priva di vita, ma eterno persiste il luogo ove sorsero le tombe della progenitrice e dei suoi antenati. A lei, a Elettra, sono dedicati i primi 18 versi, in lei si simbolizza il destino degli uomini.

Il ricorso ad Omero è costante e agisce a livelli distinti. Il cenno all' «immortal capo di Giove» è solo un primo richiamo, che presuppone l'ả $\mu \beta \rho o ́ \sigma l \alpha l ~ \delta ' \alpha ̋ \rho \alpha \chi \alpha \tilde{\imath} \tau \alpha l$

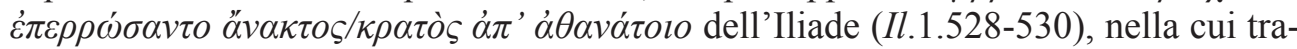
duzione Foscolo si provò nel suo Esperimento di traduzione della Iliade di Omero già ultimato nel settembre del 1806. Nell'immagine omerica del padre degli dei Foscolo introduce la nozione della liquidità, forse mutuandola dalla traduzione ritmica del poema omerico, redatta in quegli anni dal poeta italiano Melchiorre Cesarotti, che suona «le stillanti d'ambrosia auguste chiome». Dell'ambrosia, poi, elemento tradizionalmente associato alla natura divina, viene qui enfatizzata quell'efficacia magico religiosa di assimilazione alla condizione immortale e sottrazione alla corruttela mortale che si vede già operare in Omero (Il.16.670 e sgg.), allorchè Zeus, commosso dalla morte sul campo del figlio Sarpedone, ordina ad Apollo di ungerne il corpo con ambrosia e rivestirlo di vesti immortali. Foscolo riprende la funzione dell'ambrosia come elemento divinizzatore e la associa al culto della tomba: «e fe' sacro quel corpo e la sua tomba».

Quello appena menzionato è, nell'Iliade, il solo intervento diretto di Zeus nella conservazione di un corpo; dell'oceanina Elettra non si fa parola in nessuno dei due poemi. È evidente, allora, la libertà con cui Foscolo maneggia la sua fonte, trasferendo alla ninfa ciò che Zeus aveva riservato al figlio, e riuscendo così a rimodulare -all'insegna del pathos- la discendenza di Dardano.

Nei versi 244 e sgg. la figura mitica di riferimento è assolutamente la Didone virgiliana (Aen.4.317-318). Il quicquam epico viene ampliato e precisato da Foscolo con «le chiome...il viso....le dolci vigilie», così come viene raddoppiato il livello di 
subordinazione introdotto dal «se», relativo alla volontà del fato, quasi ad amplificare la distanza che divide Didone ed Elettra, destinata l'una all'abbandono, all'immortalità l'altra.

Della lunga discendenza di Dardano di matrice omerica (Il.20.215 e sgg.) Foscolo deve necessariamente omettere alcuni elementi, per evitare uno sfoggio erudito che avrebbe sovraccaricato il testo e fuorviato il lettore. Tra tutti-«Troia...Assaraco...i cinquanta talami e il regno della gente Giulia»- il poeta predilige Ilo, ovvero «l'ultimo dei principi di Ilio» (Foscolo 1807, Lettera a Monsieur Guillon. Per la sua incompetenza a giudicare i poeti italiani), cui viene attribuito un "giusto cenere ${ }^{9}$ ».

Non è un caso che del recente rinvenimento della tomba di Ilo -menzionata in vari passi omerici (Il.10.415;11.371-372;11.166;24.349) - nell'area della Troade da parte di alcuni viaggiatori, Foscolo appunti notizia in una nota al suo testo. Il sito tombale si accampa nel carme come luogo deputato alla memoria: la tripla anafora dell' «ivi» (vv. 254-255;258) segna, in un ritmo ascendente, il passaggio dal silenzio della morte («posò...dorme») al canto eterno della poesia, attraverso la profezia di Cassandra, che da pianto, «amoroso lamento», si fa canto consolatore («placando quelle afflitte alme col canto») ed eternatore («finchè il sole risplenderà sulle sciagure umane») nella figura di Omero.

Cassandra ha la funzione di contrapporre alla desolazione della distruzione di Troia il permanere dei Penati all'interno delle tombe regali e ribadire l'antinomia di apertura del carme tra materialismo e illusione di sopravvivenza: la sua divinazione si fa verità oggettiva allo stesso modo che il canto poetico si fa recupero dell'identità storica. È così che il mito si aggancia all'attualità, nel dialogo costante tra vivi e morti, che ricalca, in certo senso, le modalità stesse della relazione che Foscolo intrattiene con i classici: una dialettica lirica.

\section{LE GRAZIE}

Il rapporto con la tradizione classica - in tutti i modi che lo caratterizzarono ${ }^{10}$ trova la più ampia manifestazione ne Le Grazie, il cui progetto Foscolo avviò e riprese in un arco di tempo che va dal 1803 fino all'esilio londinese. Si tratta di un'opera che subì alterazioni di progettazione e realizzazione e che mai sfociò in un esito definitivo ${ }^{11}$; e tuttavia, in questa continua alternanza di sorti, essa è vera-

\footnotetext{
${ }^{9}$ Richiami alla memoria, il lettore, quanto detto a proposito del cinerem catulliano.

10 È interessante la classificazione di Di Benedetto (1994, passim), che parla -per le diverse fasi- di modello rovesciato, rivissuto, riverito, contaminato.

${ }^{11}$ La critica contemporanea riconosce unanimemente tre fasi essenziali nella composizione dell'opera: la prima si data al 1803, anno in cui Foscolo lavorava al commento della Chioma di Berenice, e al quale risalgono quattro frammenti poetici presentati fittiziamente come traduzioni di un antico inno greco alle Grazie. Una seconda fase, di intenso lavoro, è da collocarsi durante il soggiorno fiorentino del 1812-13, e proseguito a Milano fino al 1814: Foscolo progetta -sull'onda di suggestioni artistiche di matrice canoviana- un carme tripartito (dedicato a Venere, Vesta e Pallade), di cui comincia a elaborare frammenti sparsi; l'abbozzo di un
} 
mente illuminante per un bilancio - pure non definitivo- del legame tra il poeta e la classicità.

Ne Le Grazie, infatti, Foscolo si confronta contemporaneamente con quanto di classico il Neoclassicismo comportava e con la sua personale percezione del classico, alla luce di una intera esistenza trascorsa a cercare il senso di sé e del mondo in quello, assoluto e compiuto, degli antichi.

L'ispirazione neoclassica del Le Grazie comportava necessariamente una riflessione sul potere: già la dedica al Canova -che nel 1810 era stato invitato da Napoleone a Parigi per eseguire un ritratto dell'imperatrice Maria Luigia- si pone sulla linea di un omaggio al potere che al Foscolo doveva risultare come una forzatura; oltre a ciò, considerato che il Neoclassicismo era nato a Roma sotto la munificenza papale di Clemente XIV e Pio VI, era pressocchè impossibile pensare a forme di dissociazione del binomio arte e potere in una qualsiasi opera di stampo neoclassico.

Tuttavia, man mano che le vicende reali della storia d'Italia prendevano una direzione diversa da quella da lui desiderata (dalla delusione napoleonica già consumatasi nei Sepolcri -che si era concretizzata nella scelta di esaltare gli eroi vinti e non i vincitori del presente come invece la letteratura ufficiale rappresentata dal Monti faceva in quegli anni ${ }^{12}$ - fino alle vicende riguardanti Beauharnais, rivelatosi ben presto indegno depositario di speranze patriottiche), Foscolo inseguiva un'ispirazione che gli permettesse di ritrovare, attraverso la funzione civilizzatrice delle Grazie, valori che, riscattando il presente, lo collocassero nell'assoluto di cui solo il mito gli pareva garante.

Solo dopo il periodo fiorentino, venuta meno la visione del potere politico come punto di riferimento positivo, Foscolo potè dar voce a quei valori: «la poesia costituiva lo sbocco di un atteggiamento di ripulsa di fronte ad una realtà ostile» (Di Benedetto 1990, p.278) e Atlantide, antica isola sprofondata negli abissi del mare, diviene il luogo privilegiato per fissarli.

\section{$[\ldots]$}

85 Isola è in mezzo all'oceàn, là dove sorge più curvo agli astri; immensa terra, come è grido vetusto, un dì beata d'eterne messi e di mortali altrice. Invan la chiede all'onde oggi il nocchiero, or i nostri invocando or dell'avverso polo gli astri; e se illuso è dal desio, mira albeggiar i suoi monti da lunge,

\footnotetext{
lavoro più organico risale all'estate del 1814; il risultato è una copia in pulito che l'autore stese in quello che è noto come «Quadernone».

L'ultima fase creativa -risalente agli anni dell'esilio- consiste in un lavoro di revisione, anche questo in qualche maniera fallito, cui il poeta pose mano per curare una descrizione della collezione di opere scultoree del duca di Bedford, in cui figurava il gruppo scultoreo delle Grazie, opera di Canova: Foscolo ripropose allora la finzione della traduzione di antichi frammenti dal greco di un antico, anteponendovi una sua Dissertazione.

12 Monti era stato autore di due opere di largo successo, il Prometeo e Il Bardo della Selva Nera il cui protagonista era appunto Bonaparte.
} 
e affretta i venti, e per l'antica fama Atlantide l'appella. Ma da Febo

95 detta è Palladio Ciel, che da la santa Palla Minerva agli abitanti irata, cui il ricco suolo e gl'imenei lascivi fean pigri all'arti e sconoscenti a Giove, dentro l'Asia gli espulse, e l'aurea terra

100 cinse di ciel pervio soltanto ai Numi. Onde, qualvolta per desìo di stragi si fan guerra i mortali, e alla divina libertà danno impuri ostie di sangue; o danno a prezzo anima e brandi all'ire

105 di tiranni stranieri, o a fera impresa seguon avido re che ad innoventi popoli appresta ceppi e lutto a' suoi; allor concede le Gorgòni a Marte Pallade, e sola tien l'asta paterna

110 con che i regi precorre alla difesa delle leggi e dell'are, e per cui splende a' magnanimi eroi sacro il trionfo. Poi nell'isola sua fugge Minerva, e tutte Dee minori, a cui diè Giove

115 d'esserle care alunne, a ogni fentile studio ammaestra: e quivi casti i balli, quivi son puri i canti, e senza brina i fiori e verdi i prati, ed aureo il giorno sempre, e stellate e limpide le notti.

120 Chiamò d'intorno a sé le Dive, e a tutte compartì l'opre del promesso dono alle timide Grazie. Ognuna intenta agl'imperî correa: Pallade in mezzo con le azzurre pupille amabilmente

125 signoreggiava il suo virgineo coro. Attenuando i rai aurei del sole, volgeano i fusi nitidi tre nude Ore, e del velo distendean l'ordito. Venner le Parche di purpurei pepli

130 velate e il crin di quercia; e di più trame raggianti, adamantine, al par de l'etre e fluide e pervie e intatte mai di Morte, trame onde filan degli Dei la vita, le tre presàghe riempiean la spola. [...]

Mesci, odorosa Dea, rosee le fila; e nel mezzo del velo ardita balli,

155 canti fra'l coro delle sue speranze Giovinezza: percote a spessi tocchi 
antico un plettro il Tempo; e la danzante discende un clivo onde nessun risale. Le Grazie a' piedi suoi destano fiori,

160 a fiorir sue ghirlande: e quando il biondo crin t'abbandoni e perderai '1 tuo nome, vivran que' fiori, o Giovinezza, e intorno l'urna funerea spireranno odore. Or mesci, amabil Dea, nivee le fila;

165 e ad un lato del velo Espero sorga dal lavor di tue dita; escono errando fra l'ombre e i raggi fuor d'un mìrteo bosco due tortorelle mormorando ai baci; mirale occulto un rosignuol, e ascolta

170 silenziöoso, e poi canta imenei: fuggono quelle vereconde al bosco. Mesci, madre dei fior, lauri alle fila; e sul contrario lato erri co' specchi dell'alba il sogno; e mandi a le pupille

175 sopite del guerrier miseri i volti de la madre e del padre allor che all'are recan lagrime e voti; e quei si desta, e i prigionieri suoi guarda e sospira. Mesci, o Flora gentile, oro alle fila;

180 e il destro lembo istorïato esulti d'un festante convito: il Genio in volta prime coroni agli esuli le tazze.

Or libera è la gioia, ilare il biasmo, e candida è la lode. A parte siede

185 bello il Silenzio arguto in viso e accenna che non volino i detti oltre le soglie. Mesci cerulee, Dea, mesci le fila; e pinta il lembo estremo abbia una donna che con l'ombre e i silenzi unica veglia;

190 nutre una lampa su la culla, e teme non i vagiti del suo primo infante sien presagi di morte; e in quell'errore non manda a tutto il cielo altro che pianti. Beata! ancora non sa quanto agl'infanti 195 provido è il sonno eterno, e quÈ vagiti presagi son di dolorosa vita. (Foscolo, excerpta da Le Grazie. Inno a Pallade)

Lo scenario incantato in cui si svolge l'episodio chiave del poema -quello in cui Pallade con le Grazie e le altre dee minori attende al lavoro di tessitura del velo, protezione verso la brutalità degli uomini (bella rappresentazione plastica della funzione pedagogica dell'opera) - presenta fin dall'incipit richiami e citazioni non prive di significato. 
Contini (1986, p.158) ha evidenziato la formula omerico-virgiliana iniziale: il modulo risale infatti al libro VI dell'Odissea (vv. 244 e sgg.) in cui l'isola di Calipso, Ogigia, «giace lontano nel mare», poi ripreso nell' Eneide virgiliana (Aen.3.104 e sgg.): Creta Ious magni medio iacet insula ponto...; ed è probabilmente più il modello virgiliano a influenzare in questi versi Foscolo, per la valenza tutta positiva dell'isola in cui Anchise invita ad andare, che non l'aura negativa di quell' Ogigia in cui Ulisse piange per il mancato ritorno.

Ad Omero, ma non solo, rimanda invece il paesaggio: «.... senza brina / i fiori e verdi i prati, ed aureo il giorno / sempre, e stellate e limpide le notti». La mitezza del clima di Atlantide, se da una parte richiama l'Olimpo in cui Pallade fa ritorno in Od.6.42 e sgg., dall'altra evoca la rilettura lucreziana di quel modello, in cui le quiete sedi degli dei sono messe in relazione alla serenità fornita dalla filosofia epicurea: così Foscolo elegge Atlantide come luogo privilegiato per il superamento di uno stato di paura, ovvero per una dimensione intima e personale che renda possibile la creazione artistica attraverso la memoria poetica dei classici.

Tale ripiegamento è evidente nei versi del canto di Erato, che accompagna il lavoro della tessitrice Flora. Se l'impianto generale è desunto dal Catullo del carme LXIV relativo alle nozze di Peleo e Teti, da cui Foscolo ricava sia il dato della descrizione del tessuto che ricopre sia il modulo formale del ritornello ${ }^{13}$, le singole immagini rinviano ancora ad Omero e Lucrezio, quasi a fissare una triade di riferimento costante.

L'ago di Flora intesse le immagini che subito prendono vita - i valori che Foscolo pone alla base dell'unica armonia ormai possibile: la giovinezza, l'amore, la pietà, l'amicizia, la tenerezza materna; desunti dal mito, ma rivissuti alla luce di un pessimismo malinconico che è la misura della riconsiderazione del patrimonio classico.

Espero - menzionato in Catullo nel primo ritornello (Carm.54.329)- nei versi del Velo è invece in seconda posizione, a suggello della funzione eternatrice della poesia che vince il Tempo, che tutto vorrebbe annullare. Ed esclusivamente foscoliana è la tenerezza con cui Venere è invocata, nell'immagine di due tortorelle che fuggono vereconde verso i boschi. La distanza da Catullo è ancora più marcata nell'icona successiva, il sogno del guerriero: il poeta latino evocava le eccezionali capacità di Achille, il figlio dei due sposi. Il guerriero di Foscolo riceve nella terza strofa un sogno che ne mette a nudo l'umanità, sogno in cui i volti della madre e del padre ridestano in lui il sentimento tutto umano della pietà verso i prigionieri, e che annulla, molto più efficacemente che nei Sepolcri, l'opposizione tra vincitori e vinti: è il messaggio antibellicistico dell' Iliade che ribadisce una prospettiva intima e personale.

La gloria, anche quando negata, delle imprese militari, lascia il posto ad un convito rallegrato dal calore dell'amicizia (quarta immagine) e ad un ultimo quadro che

${ }^{13}$ Del carme catulliano prendiamo in considerazione i vv. 322 e sgg. Qui il canto per ritornelli è scandito dalla formula Currite ducentes subtegmina, currite, fusi. Foscolo escogitò una variazione che prevedeva per ogni strofa una formula parzialmente variata ma che rispettasse il modulo sintattico e fonetico: al nesso imperativo-vocativo («Mesci, ... dea») segue un complemento oggetto («fila») perfettamente corrispondente all'ultimo piede catulliano (fusi). 
dà il senso complessivo della riflessione dell'autore sulla condizione degli uomini: il pessimismo dei versi finali risulta quasi più radicale, ove si tenga conto della matrice lucreziana (LUCR. 5.218): il «vagito» del bambino appena nato, e il pianto della madre che lo considera erroneamente come «presagio di morte», sono invece segno di «dolorosa vita»; nel solco della tradizone classica del $\mu \alpha \kappa \alpha \rho \imath \sigma \mu o ́ \varsigma$ Foscolo canta «quanto agli infanti provvido è il sonno eterno». L'epitalamio catulliano è diventato così canto di morte.

È questa la risposta poetica che l'autore oppone alla realtà aggressiva ed ostile che egli non riusciva più a controllare; la disgregazione del reale impone la disgregazione del pensiero e la frammentazione formale al punto da rendere ontologicamente imperfettibile il progetto de Le Grazie: l'incompiutezza è, come sembra, l'unica misura di un'arte libera.

\section{CONCLUSIONI}

Pretendere di restituire appieno, in poche pagine, il senso di un travaglio intellettuale durato una vita intera è una operazione che richiederebbe una spregiudicatezza innecessaria, e sulla cui validità scientifica nutro forti riserve. Il senso dello studio analitico della manipolazione dei classici operata da Foscolo risiede piuttosto, a mio avviso, nelle considerazioni di ordine culturale che esso può suggerire. Il percorso che ho provato a tracciare, attraverso una selezione puntuale di testimonia, vuole denotare come la costante interlocuzione del poeta italiano con i mondi classici, greco e latino, sia il risultato di una tensione lirica. Le storie e i modi antichi non sono in Foscolo rifugio arcadico, né un'opzione negativa: il poeta parla linguaggi antichi non per vezzo estetico di gusto neoclassico, ma perché quel linguaggio è per lui il solo modo di proporre un'etica sì soggettiva ma emulabile in quanto metastorica.

Sia nella dimensione minima della dizione che in quella più ampia dell'immagine e della scena mitica, pare a me che l'esito foscoliano dell'interrogazione dei classici assuma i connotati di un rito religioso. Il poeta, cioè, ordisce una affabulazione poetica che si avvale di mitologemi, di nozioni mitiche ${ }^{14}$ tradizionali plasmandole perché siano parole efficaci e insostituibili di una cerimonia di affermazione di sé. Il ricorso al mito, per natura plastico, è un modo di ricomposizione della presenza; laddove il presente storico impedisce l'istanza etica e politica ecco che il mondo classico fornisce il poeta di quella antica arte divinatoria che è la mitopoiesi, capace di fondare una teologia, una pedagogia, un impegno civile. Foscolo «riscrive e quasi rivive la storia e la propria vicenda biografica in termini mitici» (Salvini 2003, p.72) perché il

\footnotetext{
${ }^{14}$ Entrambi i termini possono dirsi con buona approssimazione equipollenti. Tanto il primo, mitologema -nato in seno agli studi mitografici di Károly Kerény- quanto il secondo, proposto dall'antropologia di matrice sociologica del francese Louis Gernet, definiscono il nucleo minimo decifrabile, spesso iconico, di un materiale leggendario che va aggregandosi, modellandosi, alterandosi intorno ad un tema, nell'arco di una lunga diacronia.
} 
mito lo guarisce dal tempo, dal suo tempo, e il racconto classico gli offre una nuova sincronia, che definirei epico-religiosa, nella quale il poeta è eroe, non importa se vinto o vincitore. L'assimilazione eroica costituisce in ultima analisi l'assunzione di una identità che può rivolgere al presente storico una proposta culturale e morale.

Non è forse azzardato, allora, affermare che il classicismo di Foscolo sia, in fondo, un lirismo.

\section{BIBLIOGRAFIA}

\subsection{EDIZIONI:}

Bottasso, E. (1973), U. Foscolo. Poesie e prose d'arte, Torino, UTET.

Casini, T. (1891), U. Foscolo. Poesie, lettere e prose letterarie, Firenze, G.C. Sansoni.

Ferrari, S. (1891), U. Foscolo. Liriche Scelte. I Sepolcri. Le Grazie, Firenze, G.C. Sansoni. Fornaciari, R. (1897), U. Foscolo, Poesie scelte, Firenze, Barbera.

Fubini, M. (1972), Ugo Foscolo. Scritti letterari e politici. Dal 1796 al 1808, Firenze, Le Monnier.

Martinelli, D. (1987), U. Foscolo. Poesie e Sepolcri, Milano, Mondadori.

Martinetti, G.A. (1884), Dei Sepolcri. Carme di Ugo Foscolo, Torino, G.B. Paravia.

Puppo, M. (1987), U. Foscolo. Ultime lettere di Jacopo Ortis. Poesie e Carmi, Milano, Mursia.

\subsection{STUDI:}

Angrisani, M.L. (1981), «Evoluzione del tema dell'abbandono da Properzio a Foscolo» in Letterature Comparate. Problemi e metodo. Studi in onore di Ettore Paratore, Bologna, Pàtron, pp.537-548.

Binni, W. (1982), Ugo Foscolo. Storia e poesia, Torino, Einaudi.

Contini, G. (1986), Letteratura italiana del Risorgimento (1789-1861), Vol.1, Firenze, Sansoni.

Croce, B. (1950), Poesia e non poesia, Bari, Laterza.

De Camilli, D. (1986), «Eugenio di Beauharnais e Amalia Augusta di Baviera nelle Grazie del Foscolo», Italianistica 15, 267-283.

De Robertis, D. (1958), «Postilla Foscoliana», GSLI 134, 1-9.

Di Benedetto, V. (1986), Il medico e la malattia. La scienza di Ippocrate, Torino, Einaudi.

Di Benedetto, V. (1990), Lo scrittoio di Ugo Foscolo, Torino, Einaudi.

Di Benedetto, V. (1994), Nel laboratorio di Omero, Torino, Einaudi.

FischetTI, G. (1966), «L'episodio di Elettra nei Sepolcri di Foscolo», GSLI 118, 159-217.

FubIni, M. (1963), Ortis e Didimo. Ricerche e interpretazioni foscoliane, Milano, Feltrinelli. Gamberini, S. (1982), Analisi dei Sepolcri foscoliani, Messina-Firenze, D’Anna.

Marinari, A., Mineo, N. (1977), Da Foscolo all 'età della Restaurazione, Roma-Bari, Laterza. MAsinI, P.C. (1985), «Nuove pagine foscoliane», Nuova Antologia 554, 256-279. 
Mauss, M. (1950), Sociologie et Anthropologie, Paris, PUF.

Petrocchi, G. (1976), «Dalla Chioma di Berenice ai Sepolcri», Lettere Italiane 28, 304-313.

SAlvini, M. (2003) «Ugo Foscolo. Ellade patria dell'anima», in Il mito nella letteratura italiana.III, Brescia, Morcelliana, pp.60-97.

Scotti, M. (1973), Foscolo fra erudizione e poesia, Roma, Bonaci.

Segre, C. - Martignoni C. (1992), Testi nella storia, Milano, Mondadori. 